\title{
Anisotropic motion effects in CW non-linear EPR spectra: relaxation enhancement of lipid spin labels
}

\author{
V.A. Livshits, ${ }^{\mathrm{a}}$ B.G. Dzikovski, ${ }^{\mathrm{a}}$ and D. Marsh ${ }^{\mathrm{b}, *}$ \\ ${ }^{a}$ Centre of Photochemistry, Russian Academy of Sciences, 117427, Moscow, Russian Federation \\ ${ }^{\mathrm{b}}$ Max-Planck-Institut für biophysikalische Chemie, Abteilung Spektroskopie, Am Fassberg 11, D-37070 Göttingen, Germany
}

Received 5 November 2002; revised 21 February 2003

\begin{abstract}
Continuous-wave (CW) EPR measurements of enhancements in spin-lattice $\left(T_{1}-\right)$ relaxation rate find wide application for determining spin-label locations in biological systems. Often, especially in membranes, the spin-label rotational motion is anisotropic and subject to an orientational potential. We investigate here the effects of anisotropic diffusion and ordering on non-linear CWEPR methods for determining $T_{1}$ of nitroxyl spin labels. Spectral simulations are performed for progressive saturation of the conventional in-phase, first-harmonic EPR signal, and for the first-harmonic absorption EPR signals detected $90^{\circ}$-out-of-phase with respect to the Zeeman field modulation. Motional models used are either rapid rotational diffusion, or strong-jump diffusion of unrestricted frequency, within a cone of fixed maximum amplitude. Calculations of the $T_{1}$-sensitive parameters are made for both classes of $\mathrm{CW}$-experiment by using motional parameters (i.e., order parameters and correlation times), intrinsic homogeneous and inhomogeneous linewidth parameters, and spin-Hamiltonian hyperfine- and $g$-tensors, that are established from simulation of the linear CW-EPR spectra. Experimental examples are given for spin-labelled lipids in membranes.
\end{abstract}

(C) 2003 Elsevier Science (USA). All rights reserved.

\section{Introduction}

Measurement of spin-relaxation enhancements induced by the interaction of paramagnetic molecules or ions with spin labels is a proven approach to studying the spatial distribution of spin-labelled functional groups for phospholipids or proteins in membranes [14]. For reasons of sensitivity and availability, most EPR measurements on spin-labelled biomolecules are made with conventional CW instruments. Different non-linear CW-EPR techniques have been proposed to characterise the accessibility profiles for spin-labelled lipids and proteins in membranes: progressive saturation of the first-harmonic in-phase $V_{1}$-spectra [1-3], and the use of $T_{1}$-sensitive spectral parameters from the out-of-phase first- and second-harmonic $V_{1}^{\prime}$ - and $V_{2}^{\prime}$-EPR spectra [4-6]. Most studies have used saturation parameters from conventional in-phase first-harmonic $V_{1}$-EPR spectra [1-4]. These parameters, however, depend directly on rotational motion, which gives rise to line

\footnotetext{
${ }^{*}$ Corresponding author. Fax: +49-551-201-1501.

E-mail address: dmarsh@gwdg.de (D. Marsh).
}

broadening and spectral diffusion of saturation, and depend also on the intrinsic spin-spin relaxation rate. The same applies to the out-of-phase second-harmonic (saturation transfer) $V_{2}^{\prime}$-EPR spectral intensities which are sensitive to very slow molecular reorientation [6-8], and, although to a much lesser extent, to the out-ofphase first-harmonic $V_{1}^{\prime}$-EPR spectra [5,9].

The influence of isotropic rotational motion on microwave saturation behaviour, and on the $T_{1}$-sensitive parameters from out-of-phase first-harmonic EPR spectra, was analysed previously [9-11]. In membranes, however, the rotational motion of spin-labelled lipids and proteins is essentially anisotropic, and subject to an orienting potential (see, e.g., [12,13]). This results in angularly dependent transverse relaxation which, for macroscopically unoriented membranes, should lead to a distribution of saturation parameters corresponding to the different orientations of the membrane normal relative to the magnetic field. The $T_{1}$ - and $T_{2}$-relaxation enhancements of spin labels in membranes that are induced by dipolar interaction with paramagnetic ions or complexes are also angular-dependent [14]. Therefore, the influence on the saturation behaviour of the angular 
distribution in both relaxation times should also be analysed.

A number of simulation studies on linear EPR spectra have included anisotropic orientational ordering in the rotational motion [15-19]. Methods based on timedependent perturbation theory $[15,16]$ are applicable only for relatively rapid motion $\left(\tau_{\mathrm{R}} \leqslant 10^{-9} \mathrm{~s}\right)$. Those based on the stochastic Liouville equation, with spherical function expansion for the density matrix, are valid over the whole range of rotational correlation times $[17,20,21]$. They treat the overall rotational diffusion in an anisotropic medium, including an orienting potential. Recently, new methods were developed which use models of the chain dynamics for simulation of the EPR spectra [19]. Both of the latter classes of approach would be computationally extremely intensive when used for simulating non-linear EPR spectra, in the case of slow motion with restricted angular amplitude. The effects of angularly restricted Brownian diffusion on saturation transfer (out-of-phase, second-harmonic) $V_{2}^{\prime}$-EPR spectra have been analysed by Howard et al. [22], using the transition rate method. However, the influence of this motion on the $T_{1}$-sensitive parameters of the ST-EPR spectra was not considered in this work.

The aim of the present study is to develop an approach for determining spin-lattice relaxation times for anisotropically rotating spin-labelled lipids and proteins in membranes, with particular emphasis on determining spin-lattice relaxation enhancements. This is done by developing and testing simple approaches for simulating various non-linear EPR spectra (in-phase first-harmonic and out-of-phase first-harmonic EPR displays) with allowance for anisotropic rotational motion, orientational ordering, microwave saturation, and magnetic field modulation. The dependence of the $T_{1}$-sensitive parameters from these spectra on rotational frequency and angular amplitude of motion are analysed. Determination of spin-lattice relaxation enhancements is performed in two stages: (1) simulation of the corresponding linear (i.e., low-power) EPR spectra to determine both the motional parameters (order, and rotational correlation times) of the spin labels, and also the intrinsic spin-spin relaxation time $\left(T_{2}\right)$ and inhomogeneous broadening; (2) determination of the effective spin-lattice relaxation times by fitting the $T_{1}$ sensitive parameters of the simulated non-linear EPR spectra to the corresponding experimentally measured parameters, with and without a paramagnetic relaxation agent.

This approach has already been applied to progressive saturation EPR data from spin-labelled phospholipids in gel and liquid-crystalline phases of lipid membranes. This was part of an experimental study on the membrane-penetration profiles of water-soluble paramagnetic salts [14]. Here we give both the theoretical background and details of the practical implemen- tation of the simulation methods. Calculations are given for the $T_{1}$-sensitive spectral parameters which cover the practical ranges for the motional and order parameters of lipids in membranes.

\section{Materials and methods}

Spin-labelled phosphatidylcholines, $n$-PCSL (1-acyl-2[n-(4,4-dimethyloxazolidine- $N$-oxyl)stearoyl]-sn-glycero-3phosphocholine) with $n=5,8,12$, or 16 , were synthesised according to Ref. [23]. Synthetic phosphatidylcholine, DMPC (1,2-dimyristoyl-sn-glycero-3-phosphocholine) was from Avanti Polar Lipids (Alabaster, AL).

Spin-labelled phosphatidylcholines were incorporated in bilayer membranes of DMPC at a relative concentration of $1 \mathrm{~mol} \%$ by drying down the lipid solutions in chloroform and then suspending the dry lipid in water. Membrane dispersions were saturated either with argon or with air, as noted. Aliquots of the samples were loaded into $50 \mu 1,0.7 \mathrm{~mm}$ i.d., glass capillaries (Brand, Germany) and flushed with argon or air, as appropriate. Sample sizes were trimmed to $5 \mathrm{~mm}$ length to avoid inhomogeneities in the microwave $\left(H_{1}\right)$ and modulation $\left(H_{\mathrm{m}}\right)$ fields [24].

EPR spectra were recorded at a microwave frequency of $9 \mathrm{GHz}$ on a Varian Century Line or Bruker EMX spectrometer equipped with nitrogen gas flow temperature regulation. Sample capillaries were positioned along the symmetry axis of the standard $4 \mathrm{~mm}$ quartz EPR sample tube that contained light silicone oil for thermal stability. Temperature was measured with a fine-wire thermocouple located within the capillary in contact with the sample. Samples were centered in the $\mathrm{TE}_{102}$ rectangular microwave cavity and all spectra were recorded under critical coupling conditions. The rootmean-square microwave magnetic field $\left\langle H_{1}^{2}\right\rangle^{1 / 2}$ at the sample was measured as described in [24], and corrections were made for the cavity $\mathrm{Q}$ as described in the same reference. Progressive saturation measurements were made on in-phase EPR spectra recorded in the first-harmonic absorption mode ( $V_{1}$-display) at a modulation frequency of $100 \mathrm{kHz}$. First-harmonic, out-ofphase absorption EPR spectra ( $V_{1}^{\prime}$-display) were recorded as described in [5]. The modulation phase was set by the self-null method, or with a non-saturating reference sample as described in the same reference.

\section{Theory}

Two motional models are used to simulate both the low-power and non-linear EPR spectra of spin-labeled lipids in membranes: rapid tumbling in a cone and strong jump reorientation within a cone. For both models, we assume uniaxial anisotropy of the 


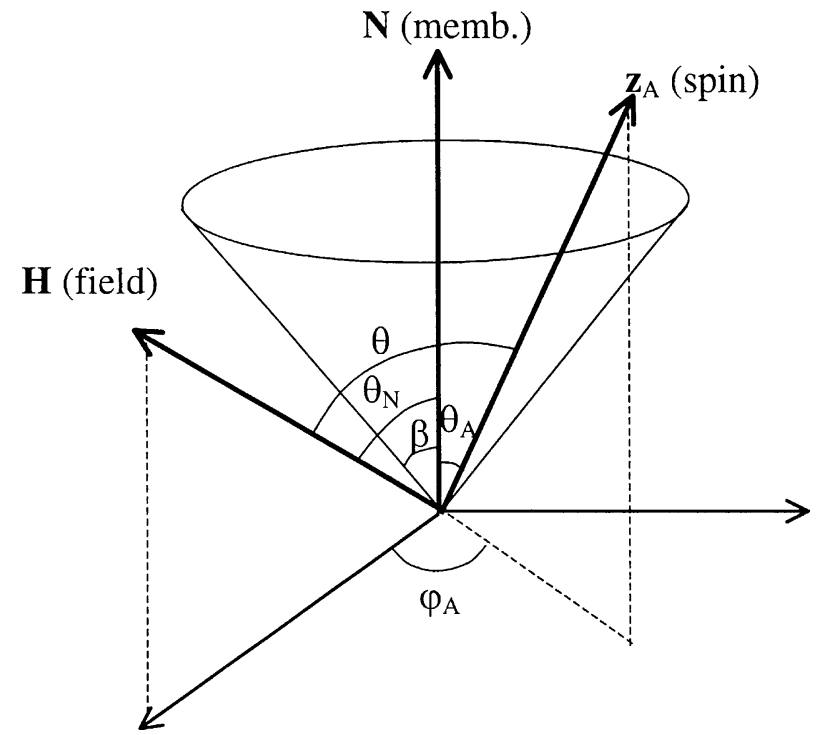

Fig. 1. Relative orientations $\left(\theta_{A}, \theta_{N}\right.$, and $\left.\theta\right)$ of the magnetic tensor symmetry axis, $\mathbf{z}_{A}$, director (i.e., membrane normal), $\mathbf{N}$, and magnetic field, $\mathbf{H}$, axes for a spin probe tumbling within a cone of semi-angle $\beta$. For convenience, the origin of $\phi_{A}$ is defined by the $\mathbf{H}-\mathbf{N}$ plane (because of uniaxial symmetry about $\mathbf{N}$ ). The trignometric relation between axis directions is given in Eq. (1).

probe environment that is characterised by the director axis (i.e., membrane normal), $\mathbf{N}$. The relations between the magnetic field direction, $\mathbf{H}$, director axis, $\mathbf{N}$, and instantaneous $z$-axis of the spin label are given in Fig. 1. The trignometrical relation between $\theta, \theta_{N}, \theta_{A}$, and $\varphi_{A}$ is

$\cos \theta=\cos \theta_{N} \cos \theta_{A}+\sin \theta_{N} \sin \theta_{A} \cos \varphi_{A}$.

For the sake of simplicity, we further suppose that tumbling inside the cone of semi-angle $\beta$ occurs with the same axial and off-axial frequency, $\tau_{\mathrm{R}}^{-1}$.

\subsection{Model 1: rapid reorientation}

Rapid tumbling in a cone results in partial averaging of the hyperfine- and $g$-tensor components by the restricted anisotropic molecular motion. The resulting resonance positions and relaxation linewidths are anisotropic and depend on the angle $\left(\theta_{N}\right)$ between the director axis and static magnetic field direction.

The resonance field position of ${ }^{14} \mathrm{~N}$ hyperfine manifold $M_{\mathrm{I}}$ is given by

$\gamma_{\mathrm{e}}\left\langle H_{\mathrm{res}}\left(\theta_{N}\right)\right\rangle=\omega_{\mathrm{L}} \frac{g_{0}}{g\left(\theta_{N}\right)}-M_{\mathrm{I}} A\left(\theta_{N}\right)$,

where $\omega_{\mathrm{L}}$ is the angular frequency of the $H_{1}$-field, $\gamma_{\mathrm{e}}$ is the electron gyromagnetic ratio and hyperfine splittings are expressed in angular frequency units. The orientation dependences of the effective $g$-value and hyperfine splitting are given by
$g\left(\theta_{N}\right)=g_{/ /} \cos ^{2} \theta_{N}+g_{\perp} \sin ^{2} \theta_{N}$,

$A\left(\theta_{N}\right)=\left(A_{/ /}^{2} \cos ^{2} \theta_{N}+A_{\perp}^{2} \sin ^{2} \theta_{N}\right)^{1 / 2}$.

The motionally averaged $g$-tensor elements in Eq. (3) are (see, e.g., [25])

$g_{\perp}=g_{0}-\frac{1}{3}\left[\Delta g \cdot S_{z z}+\delta g\left(S_{x x}-S_{y y}\right)\right]$,

$g_{/ /}=g_{0}+\frac{2}{3}\left[\Delta g \cdot S_{z z}+\delta g\left(S_{x x}-S_{y y}\right)\right]$,

and similar expressions hold for $A_{\perp}$ and $A_{/ /}$in Eq. (4), where $S_{z z}$ is the principal element of the traceless ordering tensor and: $S_{y y}=-\left(S_{z z}+S_{x x}\right)$. In terms of Cartesian spin Hamiltonian tensors, the isotropic and anisotropic parts of the $g$-tensor are defined as

$g_{0}=\frac{1}{3}\left(g_{x x}+g_{y y}+g_{z z}\right)$,

$\Delta g=g_{z z}-\frac{1}{2}\left(g_{x x}+g_{y y}\right)$,

$\delta g=\frac{1}{2}\left(g_{x x}-g_{y y}\right)$

and similar expressions for $a_{0}$ and $\Delta A$, with $\delta A \approx 0$ for a purely dipolar hyperfine anisotropy.

The order parameter of the spin-label $z$-axis is given by (cf. Fig. 1)

$S_{z z}=\frac{1}{2}\left(3\left\langle\cos ^{2} \theta_{A}\right\rangle-1\right)$

and similar expressions hold for the ordering of the molecular $x$ - and $y$-axes. For restricted random walk within a cone of semi-angle $\beta$,

$S_{z z}=\frac{1}{2} \cos \beta(1+\cos \beta)$

cf. Fig. 1. A specific model of the molecular ordering, such as this, is needed to reduce the number of parameters required for describing the linewidths.

The model used for calculating the resonance linewidths is essentially the same as that developed in [16] for simulation of linear EPR spectra. It corresponds to the second-order perturbation theory approximation: $\gamma_{\mathrm{e}} \Delta H_{\mathrm{an}} \cdot \tau_{\mathrm{R}} \ll 1$, where $\Delta H_{\mathrm{an}}$ is the anisotropy of the resonant magnetic field that is to be averaged by rotational motion in a cone, and $\tau_{\mathrm{R}}$ is the correlation time of this motion. The contribution to the transverse relaxation rate of the $M_{\mathrm{I}}$-manifold from rotational dynamics is given by (see, e.g., [16])

$$
\begin{aligned}
\frac{1}{T_{2}\left(M_{\mathrm{I}}\right)}= & {\left[\left(\omega_{\mathrm{L}} \frac{\Delta g}{g_{o}}+M_{\mathrm{I}} \Delta A\right)^{2}+\frac{3}{8}\left(I(I+1)-M_{\mathrm{I}}^{2}\right) \Delta A^{2}\right] } \\
& \times\left(\left\langle\cos ^{4} \theta\right\rangle-\left\langle\cos ^{2} \theta\right\rangle^{2}+\frac{1}{8}\left\langle\sin ^{4} \theta\right\rangle\right) \tau_{R},
\end{aligned}
$$


where angular brackets indicate averages over the rotational motion and it is assumed that axial and off-axial rotations have identical rates $\tau_{\mathrm{R}}^{-1}$. The second term in square brackets on the right of Eq. (12) is the pseudosecular contribution, with $I=1$ for ${ }^{14} \mathrm{~N}$. For spin-label reorientation restricted within a cone of semi-angle, $\beta$, the required angular averages are obtained by integrating $\theta_{A}$ from 0 to $\beta$ (see Fig. 1) using Eq. (1) [16]:

$$
\begin{aligned}
\left\langle\cos ^{4} \theta\right\rangle-\left\langle\cos ^{2} \theta\right\rangle^{2} \\
=\frac{1}{45}(1-\cos \beta)^{2}\left(4+7 \cos \beta+4 \cos ^{2} \beta\right) \\
\quad+\cos \beta \sin ^{2} \beta\left[\frac{1}{3}+\frac{2}{3} \cos \beta-\frac{1}{8}(3+5 \cos \beta) \sin ^{2} \theta_{N}\right] \\
\quad \times \sin ^{2} \theta_{N}
\end{aligned}
$$

and

$$
\begin{aligned}
\left\langle\sin ^{4} \theta\right\rangle= & \frac{1}{15}(1-\cos \beta)^{2}\left(8+9 \cos \beta+3 \cos ^{2} \beta\right) \\
& +\cos \beta(1+\cos \beta) \\
& \times\left[\sin ^{2} \beta+\frac{1}{8}\left(7 \cos ^{2} \beta-3\right) \sin ^{2} \theta_{N}\right] \sin ^{2} \theta_{N},
\end{aligned}
$$

which gives the explicit dependence on the magnetic field orientation, $\theta_{N}$ (cf. Fig. 1). Effects on the linewidth of non-axial ordering are neglected.

Absorption lineshapes $V_{1,1}$ and $V_{1,1}^{\prime}$ of the non-linear first-harmonic EPR spectra are calculated by including expressions for the averaged resonance field and relaxation linewidth in the modulation-coupled Bloch equations [26]. The resulting equations for the in-phase and out-of-phase magnetisation were solved interatively, under the small modulation amplitude approximation, as done previously for the no-motion case $[5,6]$

$$
\begin{aligned}
& V_{1,1}(D)=-V_{0,0} D \frac{2\left(D^{2}+A\right)-B \omega^{\prime 2}}{\left(D^{2}+A\right)^{2}+B^{2} \omega^{\prime 2}}, \\
& V_{1,1}^{\prime}(D)=\omega^{\prime} \cdot V_{0,0} D \frac{D^{2}+A+2 B}{\left(D^{2}+A\right)^{2}+B^{2} \omega^{\prime 2}},
\end{aligned}
$$

for $V_{1}$ - and $V_{1}^{\prime}$-spectra, respectively, where

$$
\begin{aligned}
& A=1-\omega^{\prime 2}+h^{2} \frac{a+\omega^{\prime 2}}{a^{2}+\omega^{\prime 2}}, \\
& B=-2+h^{2} \frac{1-a}{a^{2}+\omega^{\prime 2}},
\end{aligned}
$$

and $a=T_{2} / T_{1}$. In Eqs. (15)-(17), angular frequencies are expressed in terms of the transverse relaxation time, $T_{2}$, i.e.,

$$
\begin{aligned}
& \omega^{\prime}=\omega_{\mathrm{m}} T_{2}, \\
& h=\gamma_{\mathrm{e}} H_{1} T_{2}, \\
& D=\left(\gamma_{\mathrm{e}} H_{\mathrm{res}}-\omega_{\mathrm{L}}\right) T_{2},
\end{aligned}
$$

where $H_{\text {res }}$ is given by Eq. (2) and the rotational contribution to $T_{2}$ by Eq. (12), both of which depend on $\theta_{N}$. The net transverse relaxation rate is given by $1 / T_{2}=1 / T_{2}^{0}+1 / T_{2}\left(M_{\mathrm{I}}\right)$, where the intrinsic Lorentzian linewidth is $\Delta H_{\mathrm{L}}=1 / \gamma_{\mathrm{e}} T_{2}^{0}$. The Zeeman modulation frequency is $\omega_{\mathrm{m}} / 2 \pi$, and the microwave frequency is $\omega_{\mathrm{L}} / 2 \pi$.

The zeroth harmonic (in-phase) solution, $V_{0,0}$, is given by the normal slow-passage absorption lineshape [6], i.e.,

$V_{0,0}=\frac{h}{1+D^{2}+h^{2} / a}$,

where $h^{2} / a$ is the slow-passage saturation parameter. Substitution of Eq. (19) in Eqs. (15) and (16) yields the first-harmonic in-phase and out-of-phase lineshapes, respectively, for a fixed magnetic field orientation. The powder lineshape from an unoriented membrane sample is finally obtained by integration over $\theta_{N}$, with $\sin \theta_{N}$ weighting.

\subsection{Model 2: strong jump orientation}

This model of random jump diffusion was employed earlier for simulating isotropic motion of spin labels $[9,11]$. The Bloch equations that explicitly include the microwave and modulation fields are supplemented by terms that take into account random jump diffusion in a cone at a rate $\tau_{\mathrm{R}}^{-1}$. Spin magnetisation components, viz., dispersion, absorption and longitudinal magnetisation $(u, v, z)$, are expanded in Fourier harmonics, $n$, of the modulation frequency, $\omega_{\mathrm{m}}$, yielding the amplitudes $u_{n}, v_{n}, z_{n}$. Solution of the resulting infinite system of coupled equations for the Fourier coefficients is performed under the small-modulation-amplitude approximation $\left(\gamma_{\mathrm{e}} H_{\mathrm{m}} T_{2}<1\right)$. Fourier coefficients of the magnetisation components are expanded in a power series, $k$, of the modulation amplitude, $H_{\mathrm{m}}$, and an iterative procedure used to obtain the corresponding amplitudes, $u_{n, k}, v_{n, k}, z_{n, k}$. This approach is described more fully in $[9,11]$, and is readily generalised for rotational diffusion in an orienting potential.

The Bloch equation matrix is given by

$$
\hat{\mathbf{A}}^{\prime}=\left(\begin{array}{ccc}
T_{2}^{-1} & \gamma_{\mathrm{e}}\left[H-H_{\text {res }}(\theta)\right] & 0 \\
-\gamma_{\mathrm{e}}\left[H-H_{\mathrm{res}}(\theta)\right] & T_{2}^{-1} & -\gamma_{\mathrm{e}} H_{1} \\
0 & \gamma_{\mathrm{e}} H_{1} & T_{1}^{-1}
\end{array}\right),
$$

where the resonant field position, $H_{\text {res }}(\theta)$, is given by Eqs. (2)-(4) with $\theta$, which specifies the magnetic field orientation relative to the spin-label axes (see Fig. 1), replacing $\theta_{N}$ and $g_{/ /}=g_{z z}, g_{\perp}=1 / 2\left(g_{x x}+g_{y y}\right)$, etc.

The Bloch equations are solved for a fixed orientation $\left(\theta_{N}\right)$ of the director relative to the magnetic field. The integral expressions $\left[\overrightarrow{\mathbf{I}}_{n}=\int \overrightarrow{\mathbf{X}}_{n, n} \mathrm{~d} \Omega \equiv\left(U_{n, n}, V_{n, n}, Z_{n, n}\right)\right]$ for the magnetisation of the zero- and first-harmonics, 
in vector form: $\overrightarrow{\mathbf{X}}_{n, n}=\left(u_{n, n}, v_{n, n}, z_{n, n}\right)$, are similar to those for isotropic rotation $[9,11]$

$\overrightarrow{\mathbf{I}}_{0}=\left(\hat{\mathbf{E}}-\hat{\mathbf{J}}_{0} \tau_{R}^{-1}\right)^{-1} \cdot \hat{\mathbf{J}}_{0} \cdot \overrightarrow{\mathbf{M}}_{0}$,

$\overrightarrow{\mathbf{I}}_{1}=\left(\hat{\mathbf{E}}-\hat{\mathbf{J}}_{1} \tau_{R}^{-1}\right)^{-1} \cdot \overrightarrow{\mathbf{J}}_{1,0}$,

where $\mathbf{M}_{0}=M_{0}\left(0,0, T_{1}^{-1}\right)$ is the static magnetisation vector, and

$\hat{\mathbf{J}}_{n}=\int_{0}^{\beta} \int_{0}^{2 \pi} \hat{\mathbf{A}}_{n}^{-1} \sin \theta_{A} \mathrm{~d} \theta_{A} \cdot \mathrm{d} \phi_{A}$,

$\overrightarrow{\mathbf{J}}_{n, n-1}=\int_{0}^{\beta} \int_{0}^{2 \pi} \hat{\mathbf{A}}_{n}^{-1} \cdot \hat{\boldsymbol{\gamma}} \cdot \overrightarrow{\mathbf{X}}_{n-1, n-1} \sin \theta_{A} \mathrm{~d} \theta_{A} \cdot \mathrm{d} \phi_{A}$

with

$\hat{\mathbf{A}}_{n}=\hat{\mathbf{A}}^{\prime}+\left(\tau_{R}^{-1}-\mathrm{i} n \omega_{\mathrm{m}}\right) \hat{\mathbf{E}}$,

where $\hat{\mathbf{A}}^{\prime}$ is the Bloch matrix given by Eq. (20); and the gyromagnetic ratio matrix is

$\hat{\gamma}=\left(\begin{array}{ccc}0 & -\gamma_{\mathrm{e}} & 0 \\ \gamma_{\mathrm{e}} & 0 & 0 \\ 0 & 0 & 0\end{array}\right)$.

The zero-order coefficients of the spin-magnetisation vector that are required for the $\overrightarrow{\mathbf{J}}_{1,0}$ integral are [11]

$\overrightarrow{\mathbf{X}}_{0,0}=\hat{\mathbf{A}}_{0}^{-1}\left(\hat{\mathbf{E}}-\mathbf{J}_{0} \tau_{R}^{-1}\right)^{-1} \mathbf{M}_{0}$.

The integration in Eqs. (23) and (24) represents motion of the spin-label $z$-axis about the director, and is carried out over $\theta_{A}$ from 0 to $\beta$ and over $\phi_{A}$ from 0 to $2 \pi$, by substituting for $\theta$ from Eq. (1) (see also Fig. 1). All integrals depend on the angle $\theta_{N}$, which defines the orientation of the director relative to the constant magnetic field. The lineshapes of the first-harmonic in-phase and out-of-phase absorption spectra for a macroscopically unoriented sample are obtained by summing $\operatorname{Re}\left\{V_{1,1}\right\}$ and $\operatorname{Im}\left\{V_{1,1}\right\}$, respectively, over $\theta_{N}$ with $\sin \theta_{N}$ weighting, where $\overrightarrow{\mathbf{I}}_{1}=\left(U_{1,1}, V_{1,1}, Z_{1,1}\right)$.

Inhomogeneous line broadening due to hyperfine interaction with methyl and environmental protons is taken into account by convoluting this lineshape with a Gaussian function of width $\Delta H_{\mathrm{G}}$.

\section{Results and discussion}

We first present results obtained by simulation of the linear spin-label EPR spectra recorded at low microwave power. This is followed by a simulation study of the effects of anisotropic ordering and motion on progressive saturation $V_{1}$-EPR measurements, and on the intensities of first-harmonic, out-of-phase, rapid-passage $V_{1}^{\prime}$-EPR spectra. Finally, results are given on the application of the simulation methods to determine $T_{1}$-relaxation enhancements of spin-labelled lipids in membranes.

\subsection{Linear EPR spectra}

Model 1. In this model, ordering $\left(S_{x x}, S_{y y}\right)$ of the perpendicular axes $(x, y)$ relative to director, and inhomogeneous Gaussian broadening, were additionally taken into account. Also, the pseudosecular contribution to the linewidth [16] was needed for some spin labels, in order to obtain satisfactory fits.

Experimental and best-simulated EPR spectra of 5PCSL, 8-PCSL, 12-PCSL, and 16-PCSL spin-labelled lipids in fluid DMPC bilayers are presented in Fig. 2a. The lineshapes and relative intensities of the $M_{\mathrm{I}}= \pm 1$ components were fitted first because these are most sensitive to the motional parameters, rotational frequency $\left(\tau_{\mathrm{R}}^{-1}\right)$ and cone angle $(\beta)$, and to the homogeneous broadening, $\Delta H_{\mathrm{L}}$. All of these contribute to the effective spin-spin relaxation time $\left(T_{2}^{\text {eff }}\right)$ in non-linear spectra. Also, detailed simulations of the outer wings of the $M_{\mathrm{I}}= \pm 1$ components were performed to determine the relative contributions of the Lorentzian $\left(\Delta H_{\mathrm{L}}\right)$ and Gaussian $\left(\Delta H_{\mathrm{G}}\right)$ line broadening. It is seen that the agreement with experimental spectra is, in general, satisfactory but not perfect. For 5-PCSL and 8-PCSL, the problem is that to get satisfactory fits for the $M_{\mathrm{I}}= \pm 1$ components one should increase the residual $g$-value anisotropy, and this results in over-broadening the central components. These discrepancies appear to be caused by slow-motional effects which are not allowed for in the simulation model 1 .

The agreement between experimental and simulated spectra is somewhat better for 12-PCSL and 16-PCSL (see Fig. 2a). However, for 12-PCSL, the outer wing of the low-field spectral component remains more broadened than for the spectrum simulated with a purely Lorentzian intrinsic linewidth (Fig. 2a). The super-Lorentzian character of the experimental linewidth for this spin label is also probably due to slow-motional effects [27]. For 16-PCSL, a satisfactory agreement is obtained when the pseudosecular contribution to the rotational linewidth is taken into account. For 16-PCSL, the relative intensities of the $M_{\mathrm{I}}= \pm 1$ and $M_{\mathrm{I}}=0$ components are indicative of anisotropic rotational diffusion in the molecular frame, i.e., faster rotation around the $x$-axis compared with $y$ - and $z$-axes. This type of anisotropy is not allowed for in the simulation program, which explains the not-quite-satisfactory agreement with experiment. One notes, however, that for 16-PCSL the value of $T_{2}$ obtained from simulations is rather close to the phase memory time, $T_{\mathrm{m}}$, which was measured for this spin label in DMPC membranes by the spin-echo method [28].

Model 2. Simulations of the same EPR spectra of 5PCSL, 8-PCSL, 12-PCSL, and 16-PCSL performed with model 2 are presented in Fig. 2b. The fits obtained with the latter model are somewhat worse than those with model 1. Although this model is not restricted to 



Fig. 2. Experimental (solid lines) and simulated (dotted lines) spectra of the 5-PCSL, 8-PCSL, 12-PCSL, and 16-PCSL phosphatidylcholine spin labels in DMPC membranes at $39^{\circ} \mathrm{C}$. Simulations were performed (a) with model 1, and (b) with model 2 . The corresponding dynamic and linewidth parameters are given in Table 1 .

the rapid motions, it is approximate because of the adiabatic approximation and jump-like character of the molecular rotation [27]. For 16-PCSL, both models give almost identical fits. This is because, in the rapid motion region, the lineshape of the EPR spectrum is not sensitive to the rotational mechanism.

One notes that, in spite of the different lineshapes of the simulated spectra, the dynamical parameters and intrinsic linewidths obtained with model 2 are rather close to those for model 1 (see Table 1). It should also be noted that, when the spectral fits are not ideal, one can get fits with similar rms deviation by using different combinations of the parameters $\Delta H_{\mathrm{L}}, \Delta H_{\mathrm{G}}, \tau_{\mathrm{R}}^{-1}$, and $\beta$, even in terms of the same model. Thus, one can generate similar simulated spectra by decreasing (increasing) $\Delta H_{\mathrm{L}}$ by up to $0.5 \mathrm{G}$ and correspondingly decreasing (increasing) $\tau_{\mathrm{R}}^{-1}$ and increasing (decreasing) $\Delta H_{\mathrm{G}}$. A similar degeneracy in simulated $9-\mathrm{GHz}$ spectra was noted for more rigorous simulation models (see, e.g., [28]).

This uncertainty in the motional and linewidth parameters obtained from simulations of the linear EPR spectra raises the question of how this would affect the determination of relaxation parameters, and in particular, spin-lattice relaxation enhancements, from nonlinear EPR spectra. This problem is addressed in the next section by simulating non-linear EPR spectra, and in the experimental section, when determining relaxation enhancements from the $T_{1}$-sensitive parameters of the partially saturated in-phase and out-of-phase EPR spectra.

\subsection{Non-linear EPR spectra: progressive $C W$ saturation}

Using the two simulation models from above, we consider the effects of anisotropic rotation and intrinsic linewidth on the $T_{1}$-sensitive spectral parameters that are derived from the different non-linear EPR displays. Saturation of the in-phase $V_{1}$-EPR spectra is considered first, followed by consideration of the out-of-phase intensities of rapid-passage $V_{1}^{\prime}$-EPR spectra. Most simulations are carried out with model 2 in order to explore a wide range of rotational rates. In the appropriate correlation time range, simulations are also performed with model 1, with axial ordering tensor.

First-harmonic, in-phase EPR spectra were simulated for different values of the motional parameters, $\beta$ and $\tau_{\mathrm{R}}^{-1}$, the intrinsic transverse relaxation time, $T_{2}$ (or spinpacket width, $\left.\Delta H_{\mathrm{L}}=1 / \gamma_{\mathrm{e}} T_{2}\right)$, the inhomogeneous broadening, $\Delta H_{\mathrm{G}}$, and the spin-lattice relaxation time, $T_{1}$. Microwave saturation and Zeeman field modulation were included explicitly, and the motional model was that of jump rotation in a cone (specifically model 2 ). The intrinsic linewidth, $\Delta H_{\mathrm{L}}$, was varied from $3 \mathrm{G}$ down to low values $(0.25 \mathrm{G})$ at which the relative contribution of anisotropic rotational diffusion to the linewidth becomes important. 
Table 1

Motional parameters $\left(\beta, S_{y y}, \tau_{\mathrm{R}}^{-1}\right)$, intrinsic linewidths $\left(\Delta H_{\mathrm{L}}, \Delta H_{\mathrm{G}}\right)$, and spin-Hamiltonian tensors $\left(A_{i i}, g_{i i}\right)$, for spin-labelled phosphatidylcholine $n$-PCSL in dimyristoyl phosphatidylcholine membranes at $39^{\circ} \mathrm{C}$, obtained from linear EPR simulations with models 1 and 2 (see Fig. $2 \mathrm{a}$ and b)

\begin{tabular}{|c|c|c|c|c|c|c|c|c|}
\hline Spin label & Model & $\beta\left({ }^{\circ}\right)$ & $S_{y y}$ & $\tau_{\mathrm{R}}^{-1}\left(\times 10^{-8} \mathrm{~s}^{-1}\right)$ & $\Delta H_{\mathrm{L}}(\mathrm{G})$ & $\Delta H_{\mathrm{G}}(\mathrm{G})$ & $A_{i i}(\mathrm{G})^{\mathrm{a}}$ & $g_{i i}{ }^{\mathrm{a}}$ \\
\hline \multirow[t]{6}{*}{ 5-PCSL } & 1 & 54 & -0.15 & 5.5 & 1.2 & 1.2 & 5.93 & 2.0087 \\
\hline & & & & & & & 5.93 & 2.0059 \\
\hline & & & & & & & 33.73 & 2.0027 \\
\hline & 2 & 53 & - & 5.5 & $1.3^{\mathrm{b}}$ & 0.7 & 6.36 & 2.0087 \\
\hline & & & & & & & 6.36 & 2.0058 \\
\hline & & & & & & & 32.9 & 2.0029 \\
\hline \multirow[t]{6}{*}{ 8-PCSL } & 1 & 61 & -0.18 & 7 & 1.9 & 1.0 & 6.23 & 2.0088 \\
\hline & & & & & & & 6.23 & 2.0058 \\
\hline & & & & & & & 33.13 & 2.0028 \\
\hline & 2 & 60 & - & 6.5 & 1.9 & 0.7 & 6.23 & 2.0088 \\
\hline & & & & & & & 6.23 & 2.0058 \\
\hline & & & & & & & 33.23 & 2.0028 \\
\hline \multirow[t]{6}{*}{ 12-PCSL } & 1 & 80 & 0.14 & 9 & 1.2 & 0 & 5.7 & 2.0088 \\
\hline & & & & & & & 5.7 & 2.0058 \\
\hline & & & & & & & 31.7 & 2.0028 \\
\hline & 2 & 81 & - & 7.5 & 1.4 & 0 & 5.7 & 2.0092 \\
\hline & & & & & & & 5.7 & 2.0060 \\
\hline & & & & & & & 31.7 & 2.0026 \\
\hline \multirow[t]{6}{*}{ 16-PCSL } & 1 & 90 & 0.1 & 16 & 0.23 & 0.7 & 5.73 & 2.0088 \\
\hline & & & & & & & 5.73 & 2.0060 \\
\hline & & & & & & & 31.73 & 2.0028 \\
\hline & 2 & 90 & - & 16 & $0.25^{\mathrm{c}}$ & 0.7 & 5.73 & 2.0088 \\
\hline & & & & & & & 5.73 & 2.0060 \\
\hline & & & & & & & 31.73 & 2.0028 \\
\hline
\end{tabular}

\footnotetext{
${ }^{\mathrm{a}}$ Diagonal tensor values are listed in order: $x, y, z$.

${ }^{\mathrm{b}}$ Angular dependent intrinsic linewidth was used: $\Delta H_{\mathrm{L}}=\Delta H_{\mathrm{L}, 0}+\Delta H_{\mathrm{L}, 1} \cdot \sin ^{2} \theta_{0} ; \Delta H_{\mathrm{L}, 0}=1.3 \mathrm{G}, \Delta H_{\mathrm{L}, 1}=0.6 \mathrm{G}$.

${ }^{\mathrm{c}}$ Pseudosecular contribution to the linewidth was added to the intrinsic linewidth.
}

\subsubsection{Second integrals of the first-harmonic, in-phase EPR spectra}

Over the whole range of cone angles and rotational frequencies, the power saturation curves for the doubleintegrated intensities of the simulated first-harmonic spectra were found to be fitted satisfactorily by the expression

$S\left(H_{1}\right) / S\left(H_{1,0}\right)=k \cdot H_{1} / \sqrt{1+P \cdot H_{1}^{2}}$,

which was shown earlier to be valid for isotropic rotation [11]. The fitting parameter $k$ was close to $1 / H_{1,0}$ in all cases, where $H_{1,0}$ is the microwave field amplitude corresponding to the absence of saturation. On the other hand, the saturation factor, $P$, was dependent not only on $T_{1}$ and $T_{2}$, but also on the motional parameters $\beta$ and $\tau_{\mathrm{R}}^{-1}$. Because the functional dependence given by Eq. (28) corresponds to that expected for slow-passage and the no-motion case, the saturation parameter can be represented analogously as

$P=\gamma_{\mathrm{e}}^{2}\left(T_{1} T_{2}\right)^{\mathrm{eff}}$,

where $\left(T_{1} T_{2}\right)^{\text {eff }}$ is an effective $T_{1} T_{2}$-relaxation time product.
As for isotropic rotation [11], we find that the dependence of the saturation parameter on spin-lattice relaxation time $T_{1}$ is quite satisfactorily described by the linear relation

$1 / P=a+b / T_{1}$

for the entire range of values of $\beta$ and $\tau_{\mathrm{R}}^{-1}$, as is illustrated in Fig. 3. This important result shows that changes in the reciprocal saturation parameter, $\Delta(1 / P)$, are directly proportional to the spin-lattice relaxation enhancement, $\Delta\left(1 / T_{1}\right)$, for fixed values of $\beta, \tau_{\mathrm{R}}^{-1}$, and $\Delta H_{\mathrm{L}}$. One notes that, unlike the slow-passage and nomotion result given by Eq. (29), the relation between $1 / P$ and $1 / T_{1}$ contains an additional constant term, $a$, which, however, is actually rather small in most cases (see Fig. 3). The dependence on $T_{2}^{-1}$ and rotational motion of the spin label is contained in the slope, $b$, which therefore can be associated with an effective transverse relaxation rate, $1 / T_{2}^{\text {eff }}$. It should be noted, however, that $b$ and $1 / T_{2}^{\text {eff }}$ depend on the passage conditions (Zeeman modulation frequency and amplitude).

Essentially identical results, i.e., the validity of Eqs. (28) and (30), are obtained with the model of rapid tumbling in a cone (i.e., model 1) (data not shown). However, because model 2 applies to the entire range of 


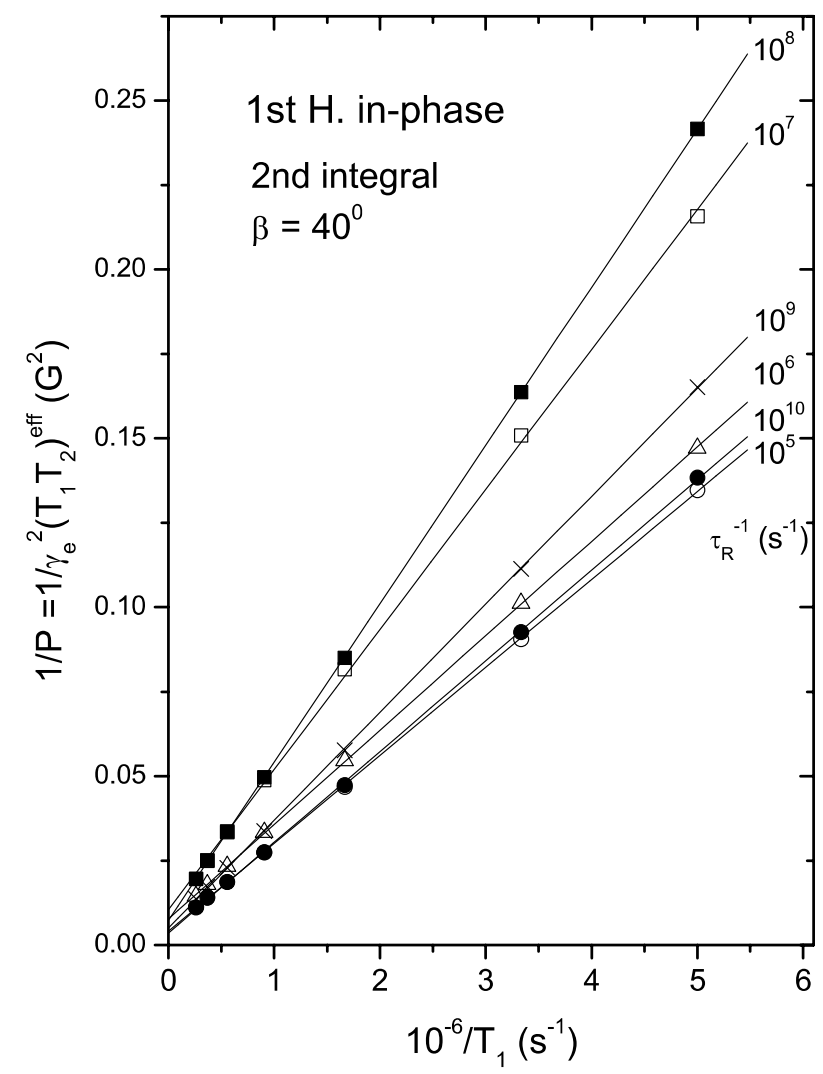

Fig. 3. Dependence of the reciprocal saturation parameter, $1 / P=1 / \gamma_{\mathrm{e}}^{2}\left(T_{1} T_{2}\right)^{\text {eff }}$, on the spin-lattice relaxation rate, $1 / T_{1}$, for different rotational frequencies, $\tau_{\mathrm{R}}^{-1}$, within a cone angle of $40^{\circ}$. Values of $P$ were determined from the second integrals of the simulated in-phase first-harmonic EPR spectra. Intrinsic linewidth is $\Delta H_{\mathrm{L}}=1.5 \mathrm{G}$, and modulation frequency $\omega_{\mathrm{m}} / 2 \pi=100 \mathrm{kHz}$. Lines are linear regressions to Eq. (30).

rotational frequencies, we used it, rather than model 1 , to study the dependence of saturation on rotational frequency and cone angle. Fig. 4 gives the dependence of the saturation parameter on rotational frequency for different rotational amplitudes. As seen from Fig. 4, $P$ decreases with increasing cone angle $\beta$. With varying rotational rate, $P$ increases about a minimum in the range $10^{7}<\tau_{\mathrm{R}}^{-1}<10^{8} \mathrm{~s}^{-1}$, on one side for progressively slower $\left(\tau_{\mathrm{R}}^{-1} \leqslant 10^{6} \mathrm{~s}^{-1}\right)$ and on the other side for more rapid $\left(\tau_{\mathrm{R}}^{-1} \leqslant 10^{9} \mathrm{~s}^{-1}\right)$ motions, at all values of $\beta$. These changes in saturation factor are related to the effective spin-spin relaxation time, $T_{2}^{\text {eff }}$ (cf. Eq. (29)). In the highfrequency (fast-motional) region, $P$ decreases with increasing $\beta$ and decreasing $\tau_{\mathrm{R}}^{-1}$, owing to the motional contribution to the effective spin-spin relaxation rate, $1 / T_{2}^{\text {eff }}$. This effect is directly proportional to the square of the anisotropy of the resonant field $\left(\Delta H_{\mathrm{an}}^{2}\right)$ and to $\tau_{\mathrm{R}}$ $[15,16]$. In the low-frequency (slow-motional) region, rotation-induced spectral diffusion leads to a decrease in microwave saturation at the resonant field. The latter effect increases with an increase in the spectral diffusion rate, which is proportional to $\Delta H_{\mathrm{an}}^{2} \tau_{\mathrm{R}}^{-1}$ (see, e.g., [20]).

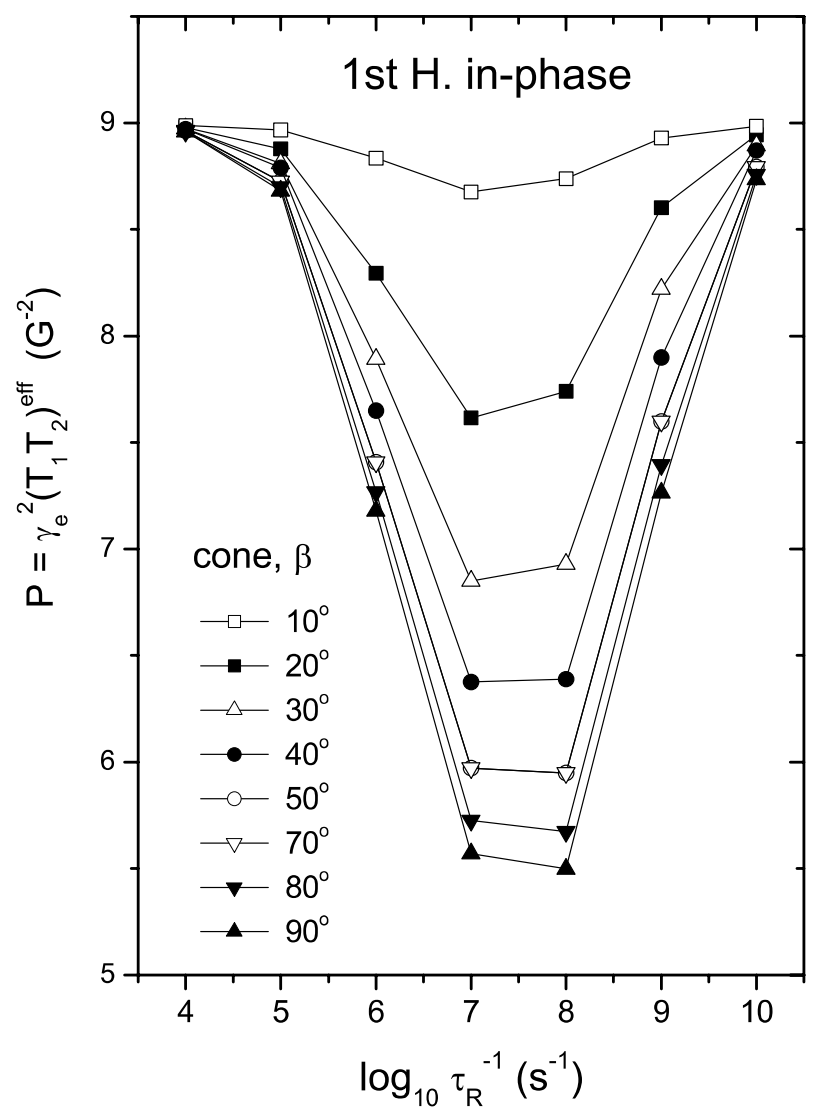

Fig. 4. Dependence of the saturation parameter, $P=\gamma_{\mathrm{e}}^{2}\left(T_{1} T_{2}\right)^{\mathrm{eff}}$, on rotational frequency, $\tau_{\mathrm{R}}^{-1}$, for different angular amplitudes, $\beta$, of the rotational motion. Values of $P$ were determined from the second integrals of the simulated in-phase, first-harmonic EPR spectra. Intrinsic linewidth is $\Delta H_{\mathrm{L}}=3 \mathrm{G}$, and $T_{1}=1.8 \mu \mathrm{s} ; \omega_{\mathrm{m}} / 2 \pi=100 \mathrm{kHz}$.

\subsubsection{Amplitudes of the first-harmonic in-phase EPR spectra}

Use of the spectral double integrals allows a direct description of the saturation curves in that the exponent $(\varepsilon=1 / 2)$ of the term containing the saturation parameter depends neither on $T_{1}$ nor on inhomogeneous broadening. Hence, this procedure gives satisfactory fits to Eq. (28) for all values of $T_{2}$ and motional parameters (cf. [11,29]). However, in cases of low signalto-noise, double integration can pose baseline problems and use of the spectral amplitudes may become necessary.

Even under slow passage conditions and in the absence of molecular motion, the saturation behaviour of the spectral lineheight, $A$, depends on the degree of inhomogeneous broadening [30]

$A\left(H_{1}\right) / A\left(H_{1,0}\right)=k \cdot H_{1} /\left(1+P \cdot H_{1}^{2}\right)^{\varepsilon}$,

where the exponent, $\varepsilon$, ranges from 1.5 to 0.5 on going from purely homogeneous broadening to overwhelmingly inhomogeneous broadening. With $\varepsilon$ as an adjustable parameter that depends additionally on the 
rotational rate, Eq. (31) also describes the power saturation curves of spin labels undergoing isotropic rotational diffusion [10].

Saturation curves for the spectral amplitudes, $A\left(H_{1}\right)$, were generated using the model of rapid tumbling in a cone (i.e., model 1 with axial order tensor) and fitted to Eq. (31). In all cases, saturation curves for the amplitudes of the low-field $\left(M_{\mathrm{I}}=+1\right)$ and central $\left(M_{\mathrm{I}}=0\right)$ simulated spectral components can be fitted satisfactorily with Eq. (31). As for the double integrals (cf. Eq. (28)), the parameter $k$ is close to $1 / H_{1,0}$, where $H_{1,0}$ is a low microwave field intensity in the linear (i.e., sub-saturating) region. The exponents, $\varepsilon$, for both low-field and central components depend on $\beta$ and $\tau_{\mathrm{R}}$, as well as on $\Delta H_{\mathrm{L}}$ and Gaussian inhomogeneous broadening (see Table 2).

The values of $\varepsilon$ given in Table 2 differ considerably from those corresponding to slow passage and no motion. In particular, for predominantly inhomogeneous broadening $\left(\Delta H_{\mathrm{L}} / \Delta H_{\mathrm{G}} \cong 0.1\right)$ the exponent is $\varepsilon \approx 1$, whereas it should be close to 0.5 in the slow passage and no-motion case when $\Delta H_{\mathrm{L}} \ll \Delta H_{\mathrm{G}}$. Furthermore, for $\Delta H_{\mathrm{L}}>\Delta H_{\mathrm{G}}$, the values of $\varepsilon$ can be anomalously high (1.6-1.9). A less satisfactory result is that $\varepsilon$ also is weakly dependent on $T_{1}$, increasing with an increase in $T_{1}$ when the motional and linewidth parameters are fixed. This is observed both for homogeneously and inhomogeneously broadened spectra (see Table 2). In general, the $T_{1}$-dependence of $\varepsilon$ is weaker for the low-field component than for the central component.

Fig. 5 shows the dependence on spin-lattice relaxation rate, $1 / T_{1}$, of the reciprocal saturation parameter, $1 / P$. Values of the latter were determined from fitting the saturation curves for the low-field $\left(M_{\mathrm{I}}=+1\right)$ and central $\left(M_{\mathrm{I}}=0\right)$ amplitudes of nitroxide spectra simulated with anisotropic rotation and molecular order (specifically model 1 with axial order tensor). Open symbols correspond to saturation curves fitted by Eq. (31) with independently variable values of $\varepsilon$, for each value of $T_{1}$. Remarkably, the linear relation that is given

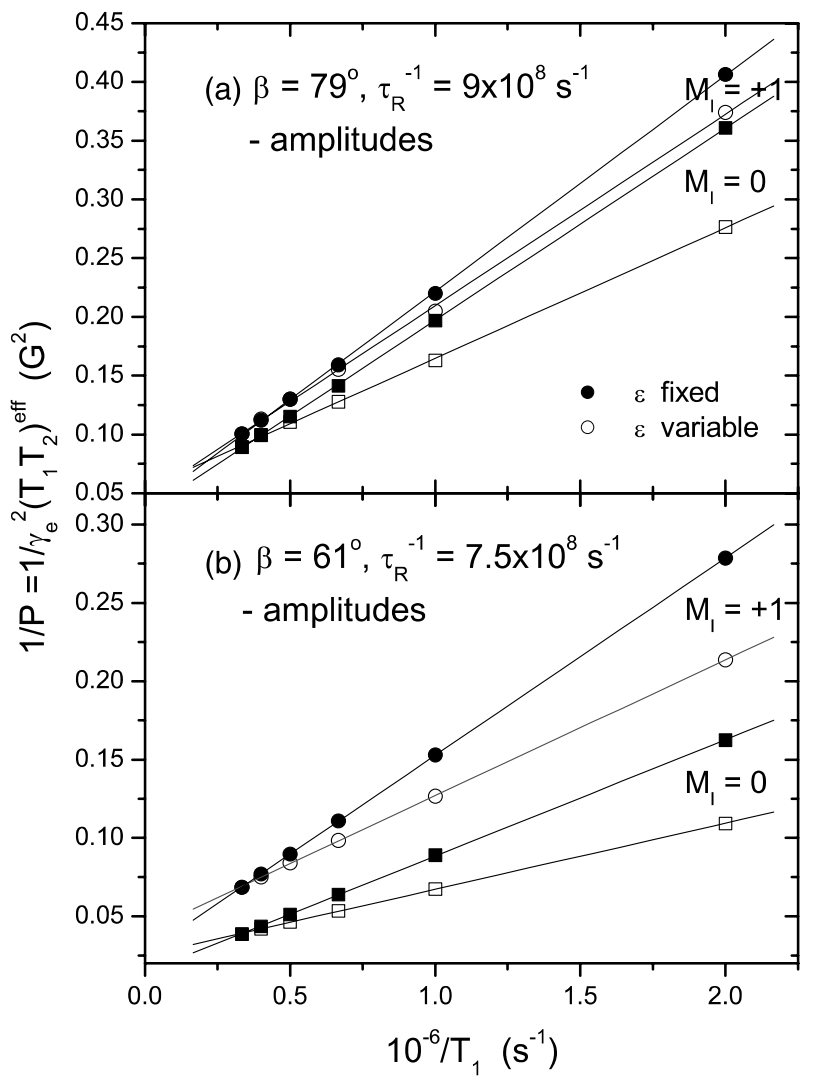

Fig. 5. Dependence of the reciprocal saturation parameter, $1 / P=1 / \gamma_{\mathrm{e}}^{2}\left(T_{1} T_{2}\right)^{\text {eff }}$, on spin-lattice relaxation rate, $1 / T_{1}$, for different motional and linewidth parameters: (a) $\beta=79^{\circ}, \tau_{\mathrm{R}}^{-1}=9 \times 10^{8} \mathrm{~s}^{-1}$, $\Delta H_{\mathrm{L}}=2 \mathrm{G} ;$ (b) $\beta=61^{\circ}, \tau_{\mathrm{R}}^{-1}=7.5 \times 10^{8} \mathrm{~s}^{-1}, \Delta H_{\mathrm{L}}=0.5 \mathrm{G}, \Delta H_{\mathrm{G}}=$ $2.0 \mathrm{G} ; \omega_{\mathrm{m}} / 2 \pi=100 \mathrm{kHz}$. Values of $P$ were determined from the saturation curves for the amplitudes of the $M_{\mathrm{I}}=+1$ (circles) and $M_{\mathrm{I}}=0$ (squares) components of the simulated in-phase, first-harmonic EPR spectra. Solid and open symbols correspond to fixed and variable exponent values $(\varepsilon)$, respectively, used in fitting the saturation curves according to Eq. (31). Lines are linear regressions to Eq. (30).

by Eq. (30) is fulfilled quite satisfactorily for both spectral amplitudes. This means that experimental values of $1 / P$, obtained in a similar way, can be used

Table 2

Dependence of the exponent, $\varepsilon$, in Eq. (31) for the saturation curves of the central $\left(M_{\mathrm{I}}=0\right)$ and low-field $\left(M_{\mathrm{I}}=+1\right)$ EPR amplitudes, on the motional $\left(\beta, \tau_{\mathrm{R}}^{-1}\right)$ and spin-relaxation $\left(\Delta H_{\mathrm{L}}, T_{1}\right)$ parameters, from simulations of first-harmonic, in-phase spin-label EPR spectra

\begin{tabular}{|c|c|c|c|c|c|c|}
\hline$\beta\left({ }^{\circ}\right)$ & $\tau_{\mathrm{R}}^{-1}\left(\times 10^{-8} \mathrm{~s}^{-1}\right)$ & $\Delta H_{\mathrm{L}}(\mathrm{G})$ & $\Delta H_{\mathrm{G}}(\mathrm{G})$ & $T_{1}(\mu \mathrm{s})$ & $\varepsilon\left(M_{\mathrm{I}}=0\right)$ & $\varepsilon\left(M_{\mathrm{I}}=+1\right)$ \\
\hline 46 & 5.0 & 0.25 & 2.0 & 0.5 & 0.85 & 0.93 \\
\hline 46 & 5.0 & 0.25 & 2.0 & 2.1 & 1.0 & 1.07 \\
\hline 46 & 5.0 & 1.0 & 2.0 & 0.5 & 1.2 & 1.0 \\
\hline 46 & 5.0 & 1.0 & 2.0 & 2.1 & 1.47 & 1.36 \\
\hline 61 & 7.5 & 0.5 & 2.0 & 0.5 & 1.02 & 0.975 \\
\hline 61 & 7.5 & 0.5 & 2.0 & 2.0 & 1.21 & 1.12 \\
\hline 61 & 7.5 & 0.5 & 2.0 & 3.0 & 1.265 & 1.156 \\
\hline 79 & 9.0 & 2.0 & 0.0 & 0.5 & 1.63 & 1.5 \\
\hline 79 & 9.0 & 2.0 & 0.0 & 2.0 & 1.91 & 1.63 \\
\hline 79 & 9.0 & 2.0 & 0.0 & 3.0 & 1.97 & 1.65 \\
\hline 79 & 9.0 & 0.5 & 2.0 & 0.5 & 1.06 & 1.02 \\
\hline 79 & 9.0 & 0.5 & 2.0 & 1.0 & 1.15 & 1.28 \\
\hline 79 & 9.0 & 0.5 & 2.0 & 2.5 & 1.28 & 1.4 \\
\hline
\end{tabular}


reliably for monitoring spin-lattice relaxation enhancements, in spite of the dependence of $\varepsilon$ on $T_{1}$.

Even if $\varepsilon$ is constrained to remain constant, the linear relation between $1 / P$ and $1 / T_{1}$ is preserved, although with different intercept and gradient. This is illustrated by the solid symbols in Fig. 5, for which $\varepsilon$ is fixed at the value obtained from the saturation curve for the longest $T_{1}$. The latter result can have important practical implications. Often, spin-label saturation in the presence of a paramagnetic relaxant decreases to such an extent that it is difficult to determine $\varepsilon$ independently from the saturation curve. Under these circumstances, one can determine $\varepsilon$ in the absence of relaxant and then use the same value in the presence of relaxant. Linear regression to the solid symbols in Fig. 5 suggests that this procedure yields acceptable results for $T_{1}$-values up to four to six times shorter than the initial values.

The relative slopes of the linear regressions in Fig. 5 indicate that the effective transverse relaxation rate, $1 / T_{2}^{\text {eff }}$, is greater for the $M_{\mathrm{I}}=+1$ hyperfine manifold than for the $M_{\mathrm{I}}=0$ manifold, as expected for motional broadening. As a rule, the averaged value of $1 / T_{2}^{\text {eff }}$ that is obtained from the saturation behaviour of the double integral lies between those for these two spectral amplitudes.

Because of the interrelated dependence of the saturation behaviour on the motional properties and transverse relaxation behaviour of the spin label, comparison of relaxation enhancements deduced from different spin labels (e.g., the various $n$-PCSL) becomes problematic. A common practice is to allow for the effects of rotational motion and intrinsic $T_{2}$ by dividing $1 / P$ by the peak-to-peak (p-p) linewidth of the central component. However, simulations show that use of this ratio only partly reduces the above dependence. In the case of strong inhomogeneous broadening, or a change from inhomogeneous to homogeneous broadening, it gives no improvement at all. For instance, for motional parameters close to those for 4-PCSL in DMPC membranes at $39^{\circ} \mathrm{C}$ (see Table 1) and inhomogeneous broadening of $\Delta H_{\mathrm{G}}=2 \mathrm{G}$, changing $\Delta H_{\mathrm{L}}$ from 0.25 to $1 \mathrm{G}$ results in a threefold increase in $1 / P$ for the central component (from 0.067 to $0.2 \mathrm{G}^{2}$, with $T_{1}=2 \mu \mathrm{s}$ ), whereas its $\mathrm{p}$-p linewidth increases by only $12 \%$.

Under these circumstances, accurate comparison of relaxation enhancements for different spin labels requires spectral simulation to achieve quantitative reliability. Our present approach involves two simulation steps. First, the motional parameters $\beta$ (or $S_{z z}$ ) and $\tau_{\mathrm{R}}^{-1}$, and linewidths $\Delta H_{\mathrm{L}}$ and $\Delta H_{\mathrm{G}}$, are determined by simulation of the low-power (linear) EPR spectra. Then these parameters are used to simulate non-linear EPR spectra and derive saturation curves for second integrals or spectral amplitudes. In the latter step, $T_{1}$ is varied to reach an agreement with the saturation parameters, $P$, and exponents, $\varepsilon$, determined from the experimental saturation curves. Application of this approach to $n$ PCSL spin labels in DMPC-membranes will be given in the section devoted to experimental results.

\subsection{Non-linear EPR spectra: out-of-phase displays}

As in the case of isotropic rotation [9], the lineshapes of the first-harmonic out-of-phase EPR spectra $\left(V_{1}^{\prime}\right)$ for anisotropic motion are similar to those of the conventional EPR spectra $\left(V_{1}\right)$, at sufficiently high values of $H_{1}$. Therefore the same $T_{1}$-sensitive spectral parameters are used as for isotropic rotation. The ratio, $\rho_{1}^{\prime}$, of second integrals of the $V_{1}^{\prime}$ and $V_{1}$ signals is defined by

$\rho_{1}^{\prime}=\iint V_{1}^{\prime}(H) \cdot \mathrm{d}^{2} H / \iint V_{1}(H) \cdot \mathrm{d}^{2} H$

and the out-of-phase/in-phase ratios of the amplitudes of the low-field $\left[\rho_{1}^{\prime}\left(M_{\mathrm{I}}=+1\right)\right]$ and central $\left[\rho_{1}^{\prime}\left(M_{\mathrm{I}}=0\right)\right]$ components are defined as

$\rho_{1}^{\prime}\left(M_{\mathrm{I}}\right)=V_{1}^{\prime}\left(M_{\mathrm{I}}\right) / V_{1}\left(M_{\mathrm{I}}\right)$

for spectral positions corresponding to the peak of the particular $M_{\mathrm{I}}$ manifold.

The $T_{1}$-dependence of these parameters was determined from first-harmonic spectra simulated for different modulation frequencies and different values of cone angle $\beta$ (or order parameter, $S_{z z}$ ), $\tau_{\mathrm{R}}^{-1}, \Delta H_{\mathrm{L}}$, and $\Delta H_{\mathrm{G}}$. At high microwave power, the form of the $T_{1}$-dependence is similar to that obtained previously for either static or isotropic motion [5,9]. Fig. 6 gives the dependence of the out-of-phase/in-phase second-integral ratio on $T_{1}$, for one case of anisotropic motion in an orienting potential. As for the static case [5], the dependence on intrinsic linewidth is not great, especially at the lower modulation frequency of $\omega_{\mathrm{m}} / 2 \pi=25 \mathrm{kHz}$. Again also, $\rho_{1}^{\prime}$ at $100 \mathrm{kHz}$ is larger and more sensitive to shorter values of $T_{1}$ than at $25 \mathrm{kHz}$ modulation frequency.

Fig. 7 gives the dependence of the $T_{1}$-sensitivity of the $\rho_{1}^{\prime}$ ratio on rotational rate, $\tau_{\mathrm{R}}^{-1}$, and cone angle, $\beta$. The dependence on $\tau_{\mathrm{R}}^{-1}$ is limited and comparable to that for isotropic motion [9]; that on angular amplitude, $\beta$, is slight, except at intermediate rotational frequencies. Fig. 8 shows the dependence of the $\rho_{1}^{\prime}$ ratio on rotational rate, $\tau_{\mathrm{R}}^{-1}$, for different values of cone angle, $\beta$, and a fixed value of $T_{1}$, at high modulation frequency $\left(\omega_{\mathrm{m}} / 2 \pi=100 \mathrm{kHz}\right)$. The functional dependence is similar to that for the saturation parameter, $P$ (cf. Fig. 4), except that quantitatively the dependence of $\rho_{1}^{\prime}$ on these parameters is much less than that of the saturation parameter.

Essentially similar simulation results to those obtained from the spectral integrals are also obtained for the out-of-phase/in-phase ratios of the first-harmonic spectral amplitudes at the $M_{\mathrm{I}}=0$ and $M_{\mathrm{I}}=+1$ positions (data not shown). Thus the $\rho_{1}^{\prime}\left(M_{\mathrm{I}}\right)$ parameters display only low sensitivity to motional parameters and a dependence on $\beta$ and $\tau_{\mathrm{R}}^{-1}$ that is similar to that in Fig. 8 . 


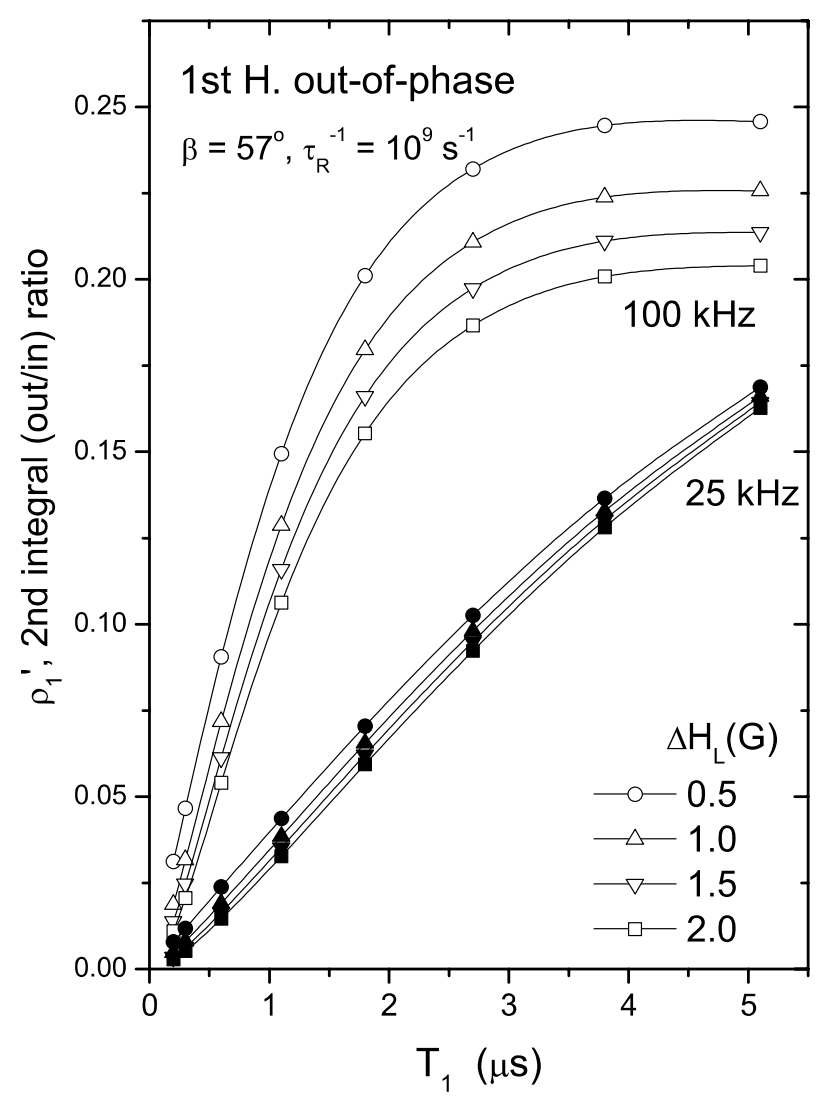

Fig. 6. Dependence on spin-lattice relaxation time, $T_{1}$, of the ratio, $\rho_{1}^{\prime}$, of the double-integrated intensities of the first-harmonic out-of-phase and in-phase EPR spectra from spin labels undergoing anisotropic rotation in an orientational potential. Spectra are simulated for Zeeman modulation frequencies, $\omega_{\mathrm{m}} / 2 \pi$, of $100 \mathrm{kHz}$ (open symbols) and $25 \mathrm{kHz}$ (solid symbols), and values of intrinsic linewidth, $\Delta H_{\mathrm{L}}$, given in the figure. Dynamic parameters: $\beta=57^{\circ}, \tau_{\mathrm{R}}^{-1}=10^{9} \mathrm{~s}^{-1} ; H_{1}=0.5 \mathrm{G}$.

The dependence of the first-harmonic, out-of-phase intensities on motional amplitude and rate is therefore less significant than that of the saturation parameters obtained from progressive saturation experiments with the in-phase spectra. Nevertheless, for greater precision in determining relaxation enhancements, corrections for these variables should be made by using the two-stage simulation strategy that was proposed in the case of progressive saturation experiments.

\subsection{Non-linear EPR: experimental applications}

Phosphatidylcholine spin labels, $n$-PCSL, with the nitroxide group at different positions in the $s n-2$ acyl chain are used as a practical example. In fluid bilayer membranes of dimyristoyl phosphatidylcholine, the $n$-PCSL EPR spectra are characteristic of different degrees of molecular ordering (i.e., rotational amplitudes) and different rotational rates (see Fig. 2). Values of $n$ are chosen to cover most of the experimentally useful range of spectral anisotropy. Effective values of $T_{1}$ and spin-

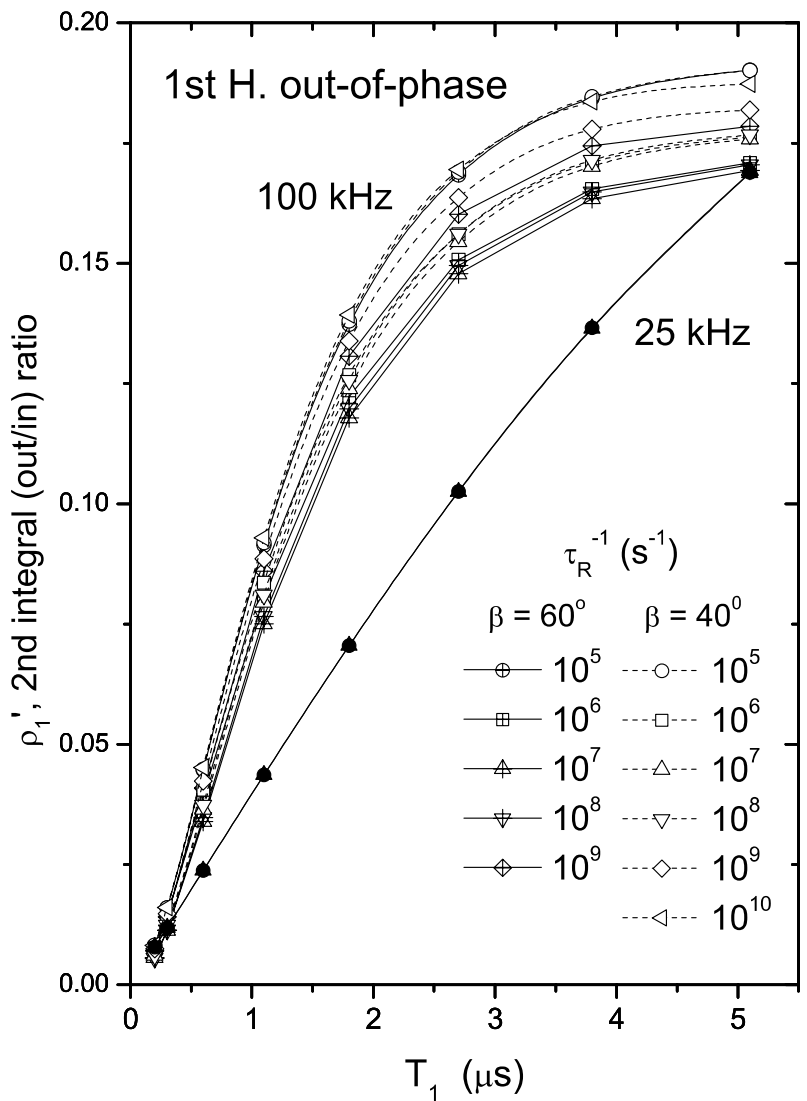

Fig. 7. Dependence on spin-lattice relaxation time, $T_{1}$, of the out-ofphase/in-phase ratio, $\rho_{1}^{\prime}$, for the double-integrated intensities from first-harmonic EPR spectra simulated for different rates $\left(\tau_{\mathrm{R}}^{-1}=10^{5}-10^{10} \mathrm{~s}^{-1}\right)$ and angular amplitudes $\left(\beta=40^{\circ}, 60^{\circ}\right)$ of rotational motion, and a Zeeman modulation frequency of $\omega_{\mathrm{m}} / 2 \pi=100 \mathrm{kHz}$ (open symbols and centred symbols). For $\omega_{\mathrm{m}} / 2 \pi=25 \mathrm{kHz}$ (solid symbols), data are given for $\tau_{\mathrm{R}}^{-1}=10^{9} \mathrm{~s}^{-1}$ and $\beta=29^{\circ}(\bullet)$ or $57^{\circ}(\boldsymbol{\Delta})$. Intrinsic linewidth $\Delta H_{\mathrm{L}}=3 \mathrm{G} ; H_{1}=0.5 \mathrm{G}$.

lattice relaxation enhancements $\Delta\left(1 / T_{1}\right)$ are obtained from simulations of the non-linear EPR spectra with the same motional models and parameters that were used for simulation of the linear spectra. The $T_{1}$-relaxation time was used as an input parameter that was varied to obtain best agreement between experimental and calculated saturation curves and out-of-phase spectral intensities.

\subsubsection{Progressive $C W$-saturation}

Saturation curves for samples saturated either with argon or with air are well described by Eq. (28), for the double integrated intensities. Similarly, the saturation curves for the spectral amplitudes conform to Eq. (31) where the exponent values, however, depend on the spin-label position. Table 3 gives the values of exponent $\varepsilon$ for various $n$-PCSL in DMPC membranes. As for the simulated saturation curves (Table 2), this variability in the experimental values of $\varepsilon$ is evidently due to changes in motional and linewidth parameters with spin label 




Fig. 8. Dependence of the out-of-phase/in-phase ratio, $\rho_{1}^{\prime}$, of the double-integrated intensities of the simulated first-harmonic EPR spectra on rotational frequency, $\tau_{\mathrm{R}}^{-1}$, for different angular amplitudes, $\beta$, of rotational motion. Intrinsic linewidth is $\Delta H_{\mathrm{L}}=3 \mathrm{G}$ and $T_{1}=1.8 \mu \mathrm{s} ; \omega_{\mathrm{m}} / 2 \pi=100 \mathrm{kHz} ; H_{1}=0.5 \mathrm{G}$.

position in the membrane. One sees from Table 3, that the values of the saturation parameter $P$ determined with Eq. (31) and variable $\varepsilon$ can differ somewhat from those determined with a fixed value of $\varepsilon=1.5$, which is appropriate to purely homogeneous linebroadening. However, it is found that the $T_{1}$-values determined selfconsistently with the two different sets of parameters are close to each other.

Application of the present simulation methods to determination of spin-lattice relaxation enhancements has already been given for $n$-PCSL spin labels in DMPC membranes [14]. Penetration profiles were established for those paramagnetic ion salts that have appreciable membrane solubility. For Ni perchlorate, the enhancement in $T_{2}$-relaxation rate determined from the homogeneous linewidth was equal to that in $T_{1}$-relaxation rate determined from progressive saturation. This agreement of the two relaxation rates, which is characteristic of the Heisenberg spin-exchange interaction, provides experimental support for the present simulation strategy. Similar experiments on the relaxation enhancement by pure oxygen, which is known to have predominantly a Heisenberg exchange mechanism [31], also reveal equality of the $T_{1}$ - and $T_{2}$-rates when analysed with the present simulation methods [32]. Here we illustrate use of the $T_{1}$-methods to determine the weaker relaxation enhancement induced by oxygen in air.

Table 4 gives the effective values of $T_{1}$ that are obtained for the $n$-PCSL spin labels in fluid DMPC bilayers from progressive saturation measurements on the first-harmonic, in-phase $V_{1}$-EPR spectra. For samples saturated with air, determinations are made by simulating the experimental saturation curves from both the double-integrated spectral intensity and the amplitudes of the low-field $\left(M_{\mathrm{I}}=+1\right)$ and central $\left(M_{\mathrm{I}}=0\right)$ hyperfine manifolds. Values from the double integral are not given for samples saturated with argon (i.e., with long $T_{1}$ ), because the extreme saturation broadening results in truncation of the spectral integral at high powers. Model 1 (as largely appropriate to a temperature of $39^{\circ} \mathrm{C}$ ) is used for the simulations of all samples (cf. Fig. 2). Nonaxiality of the ordering tensor is included as shown in Table 1. For argon-saturated samples, model 2 is also used to derive $T_{1}$-values from the $M_{\mathrm{I}}=0$ amplitude. These values are given in parentheses in Table 4. Good agreement is found with the values obtained by using model 1. As seen from Table 4, there is a reasonable agreement between all three methods of $T_{1}$-determination, especially between those based on the $M_{\mathrm{I}}=+1$ and $M_{\mathrm{I}}=0$ amplitudes. The values of $T_{1}$ obtained from the second integrals are systematically, but not appreciably, higher. The relaxation enhancements, $\Delta\left(1 / T_{1}\right)$, induced by air at $39^{\circ} \mathrm{C}$ are: $2.55 \pm 0.05,2.8 \pm 0.1,3.40 \pm 0.3$, and $2.9 \pm 0.2 \mathrm{MHz}$ for 5-PCSL, 8-PCSL, 12-PCSL, and 16-PCSL, respectively. Corresponding values measured by saturation recovery are $2-3 \mathrm{MHz}$ at the 5-position and $3-5 \mathrm{MHz}$ at the 16-position of stearic acid, over the temperature range $29-45^{\circ} \mathrm{C}$ in DMPC [33].

Table 3

Saturation parameters, $P\left(\mathrm{G}^{-2}\right)$, and exponents, $\varepsilon$, determined from fitting the saturation curves for the amplitudes of the low-field $\left(M_{\mathrm{I}}=+1\right)$ and central $\left(M_{\mathrm{I}}=0\right)$ components to Eq. (31) for different $n$-PCSL spin labels in argon-saturated DMPC membranes at $39^{\circ} \mathrm{C}$

\begin{tabular}{clccccc}
\hline$n$-PCSL & $\varepsilon\left(M_{\mathrm{I}}=+1\right)$ & $P\left(M_{\mathrm{I}}=+1\right)$ & $P\left(M_{\mathrm{I}}=+1\right)^{\mathrm{a}} \varepsilon=1.5$ & $\varepsilon\left(M_{\mathrm{I}}=0\right)$ & $P\left(M_{\mathrm{I}}=0\right)$ & $P\left(M_{\mathrm{I}}=0\right)^{\mathrm{a}} \varepsilon=1.5$ \\
\hline 5 & 1.40 & 4.5 & 4.0 & 1.76 & 7.9 & 10.6 \\
8 & 1.71 & 3.0 & 3.7 & 1.75 & 7.6 & 10.1 \\
12 & 1.58 & 7.7 & 8.4 & 1.76 & 7.8 & 10.4 \\
16 & 1.51 & 17.7 & 17.9 & 1.46 & 16.2 & 15.3 \\
\hline
\end{tabular}

${ }^{\text {a }}$ Saturation parameters determined by fitting the saturation curves with a fixed value of $\varepsilon=1.5$ in Eq. (31). 
Table 4

Effective spin-lattice relaxation times, $T_{1}(\mu \mathrm{s})$, of $n$-PCSL spin labels in air- or argon-saturated DMPC membranes at $39^{\circ} \mathrm{C}$, determined from saturation parameters, $P$, in progressive saturation of the first-harmonic, in-phase spectral amplitudes of the low-field $\left(M_{\mathrm{I}}=+1\right)$ and central $\left(M_{\mathrm{I}}=0\right)$ components, and of the second integral; and determined from out-of-phase to in-phase ratios, $\rho_{1}^{\prime}$, of the low-field $\left(M_{\mathrm{I}}=+1\right)$ and central $\left(M_{\mathrm{I}}=0\right)$ spectral amplitudes, and second integrals, of the first-harmonic EPR spectra ${ }^{a}$

\begin{tabular}{|c|c|c|c|c|c|}
\hline \multirow[t]{2}{*}{$n$-PCSL } & \multicolumn{2}{|c|}{$M_{\mathrm{I}}=+1$} & \multicolumn{2}{|c|}{$M_{\mathrm{I}}=0$} & \multirow[t]{2}{*}{ Second integral air } \\
\hline & Air & Argon & Air & Argon & \\
\hline \multicolumn{6}{|c|}{ Saturation parameters, $P$} \\
\hline 5 & 0.30 & 1.20 & 0.31 & $1.58(1.53)$ & 0.334 \\
\hline 8 & 0.27 & 1.20 & 0.30 & $1.58(1.50)$ & 0.330 \\
\hline 12 & 0.25 & 1.05 & 0.21 & $0.99(1.00)$ & 0.253 \\
\hline 16 & 0.29 & 1.38 & 0.25 & $1.15(1.57)$ & 0.260 \\
\hline \multicolumn{6}{|c|}{ Out-of-phase ratio, $\rho_{1}^{\prime}$} \\
\hline 5 & 0.44 & 2.05 & 0.485 & 2.27 & - \\
\hline 8 & 0.43 & 2.29 & 0.46 & 2.43 & 0.51 \\
\hline 12 & 0.35 & 1.68 & 0.38 & 1.67 & 0.405 \\
\hline 16 & 0.35 & 1.55 & 0.35 & 1.58 & 0.439 \\
\hline
\end{tabular}

${ }^{\mathrm{a}} T_{1}$ values are obtained by simulating the non-linear spectra using model 1 (model 2 ).

\subsection{Out-of-phase spectra}

Effective values of $T_{1}$ determined from the ratios, $\rho_{1}^{\prime}$, of the amplitudes or second integrals of the out-of-phase to in-phase first-harmonic EPR spectra are given also in Table 4. Again, these are determined by simulation of the out-of-phase spectra, using model 1 with non-axial order tensor as indicated in Table 1. As for the progressive saturation measurements, agreement between the values determined from the different spectral parameters is satisfactory. However, the absolute values of $T_{1}$ obtained by using the out-of-phase to in-phase ratios are systematically higher than those obtained from the saturation curves. The reason for this discrepancy is not quite clear. Probably it is caused by approximations used in the simulations that are manifested differently for the two classes of measurement. For comparison with the $\rho_{1}^{\prime}\left(M_{\mathrm{I}}=0\right)$ data in Table 4 , the $T_{1}$-values measured by saturation recovery for 5-position and 16position labelled stearic acid in DMPC at $45^{\circ} \mathrm{C}$ are 3.2 and $1.8 \mu \mathrm{s}$, respectively, in the absence of oxygen [34]. Note, however, that effective $T_{1}$-values derived from $\mathrm{CW}$ measurements must not necessarily coincide with direct measurements of $T_{1 \mathrm{e}}$, because of contributions to the former from nuclear relaxation (see [11]). As already emphasised, in the CW studies we concentrate on the measurement of enhancements rather than on absolute rates of $T_{1}$-relaxation.

The relaxation enhancements, $\Delta\left(1 / T_{1}\right)$, induced by paramagnetic oxygen in air have comparable values when deduced from progressive saturation and out-ofphase intensities. From the latter measurements, $\Delta\left(1 / T_{1}\right)=1.7 \pm 0.1, \quad 1.83 \pm 0.06, \quad 2.15 \pm 0.12, \quad$ and $2.22 \pm 0.01 \mathrm{MHz}$ for 5-PCSL, 8-PCSL, 12-PCSL, and 16-PCSL, respectively. In general, the enhancements in relaxation rate deduced from saturation parameters, $P$, are somewhat larger than those deduced from the in- tensity ratio, $\rho_{1}^{\prime}$. Both show similar trends with increasing spin-label position, $n$.

\section{Conclusions}

Determinations of $T_{1}$-relaxation enhancements for spin labels from CW non-linear EPR must allow for differing rotational rates and degrees of ordering. This can be done by simulating the anisotropic non-linear spectra with motional and relaxation parameters established from simulation of the linear spectra. Relatively simple models for the restricted anisotropic motion suffice to give consistent measurements of relaxation enhancements deduced from different positions (or integrated intensities) in the anisotropic spectra. Consistency is also achieved between progressive saturation and out-of-phase non-linear methods, although systematic discrepancies can be attributed to the simplicity of the simulation models.

Such considerations are essential when determining the permeation profiles of paramagnetic species into membranes from non-linear EPR measurements on spin-labelled lipids [14]. They are also of crucial importance in determinations of nitroxide location by using site-directed spin-labelling technology [35]. If corrections for anisotropic ordering are made, as described here, greater precision can be achieved by determining separately the accessibility profiles to different relaxation agents, rather than relying upon $\Phi$ ratios from pairs of complementary relaxants [36].

\section{Acknowledgments}

We thank Frau B. Angerstein for synthesis of spinlabelled lipids. This work was supported in part by a 
collaborative grant from the Deutsche Forschungsgemeinschaft and the Russian Academy of Sciences, and by the Russian Foundation for Basic Research, grant 01-03-32232.

\section{References}

[1] G.I. Likhtenstein, A.V. Kulikov, A.I. Kotelnikov, L.A. Levchenko, Methods of physical labels - a combined approach to the study of microstructure and dynamics in biological systems, J. Biochem. Biophys. Methods 12 (1986) 1-28.

[2] C. Altenbach, T. Marti, H.G. Khorana, W.L. Hubbell, Transmembrane protein structure: spin labelling of bacteriorhodopsin mutants, Science 248 (1990) 1088-1092.

[3] W.L. Hubbell, A. Gross, R. Langen, M.A. Lietzow, Recent advances in site-directed spin labeling of proteins, Curr. Opin. Struct. Biol. 8 (1998) 649-656.

[4] D. Marsh, T. Páli, L.I. Horváth, Progressive saturation and saturation transfer EPR for measuring exchange processes and proximity relations in membranes, in: L.J. Berliner (Ed.), Spin Labeling. The Next Millenium, Plenum Press, New York, 1998, pp. 23-82.

[5] V.A. Livshits, T. Páli, D. Marsh, Spin relaxation measurements using first-harmonic out-of-phase absorption EPR signals, J. Magn. Reson. 134 (1998) 113-123.

[6] T. Páli, V.A. Livshits, D. Marsh, Dependence of saturationtransfer EPR intensities on spin-lattice relaxation, J. Magn. Reson. B 113 (1996) 151-159.

[7] D.D. Thomas, L.R. Dalton, J.S. Hyde, Rotational diffusion studied by passage saturation transfer electron paramagnetic resonance, J. Chem. Phys. 65 (1976) 3006-3024.

[8] L.I. Horváth, D. Marsh, Analysis of multicomponent saturation transfer ESR spectra using the integral method: application to membrane systems, J. Magn. Reson. 54 (1983) 363-373.

[9] V.A. Livshits, D. Marsh, Spin relaxation measurements using first-harmonic out-of-phase absorption EPR signals: rotational motion effects, J. Magn. Reson. 145 (2000) 84-94.

[10] D.A. Haas, C. Mailer, B.H. Robinson, Using nitroxide spin labels. How to obtain $T_{\mathrm{le}}$ from continuous wave electron paramagnetic resonance spectra at all rotational rates, Biophys. J. 64 (1993) 594-604.

[11] V.A. Livshits, T. Páli, D. Marsh, Relaxation time determinations by progressive saturation EPR: Effects of molecular motion and Zeeman modulation for spin labels, J. Magn. Reson. 133 (1998) 79-91.

[12] D. Marsh, Experimental methods in spin-label spectral analysis, in: L.J. Berliner, J. Reuben (Eds.), Biological Magnetic Resonance, vol. 8, Plenum, New York, 1989, pp. 255-303.

[13] D. Marsh, L.I. Horváth, Spin-label studies of the structure and dynamics of lipids and proteins in membranes, in: A.J. Hoff (Ed.), Advanced EPR. Applications in Biology and Biochemistry, Elsevier, Amsterdam, 1989, pp. 707-752.

[14] V.A. Livshits, B.G. Dzikovski, D. Marsh, Mechanism of relaxation enhancement of spin labels in membranes by paramagnetic ion salts: dependence on $3 \mathrm{~d}$ and $4 \mathrm{f}$ ions and on the anions, J. Magn. Reson. 148 (2001) 221-237.

[15] H. Schindler, J. Seelig, EPR spectra of spin labels in lipid bilayers, J. Chem. Phys. 59 (1973) 1841-1850.

[16] J. Israelachvili, J. Sjösten, L.E.G. Eriksson, M. Ehrström, A. Gräslund, A. Ehrenberg, ESR spectral analysis of the molecular motion of spin labels in lipid bilayers and membranes based on a model in terms of two angular motional parameters and rotational correlation times, Biochim. Biophys. Acta 382 (1975) 125-141.

[17] C.F. Polnaszek, G.V. Bruno, J.H. Freed, ESR line shapes in slowmotional region-anisotropic liquids, J. Chem. Phys. 58 (1973) 3185-3199.
[18] A. Lange, D. Marsh, K.-H. Wassmer, P. Meier, G. Kothe, Electron spin resonance study of phospholipid membranes employing a comprehensive line-shape model, Biochemistry 24 (1985) 4383-4392.

[19] R. Cassol, M.T. Ge, A. Ferrarini, J.H. Freed, Chain dynamics and the simulation of electron spin resonance spectra from oriented phospholipid membranes, J. Phys. Chem. B 101 (1997) 8782-8789.

[20] J.H. Freed, Theory of slowly tumbling ESR spectra for nitroxides, in: L.J. Berliner (Ed.), Spin Labeling, Theory and Applications, Academic Press, New York, 1976, pp. 53-132.

[21] M. Moser, D. Marsh, P. Meier, K.-H. Wassmer, G. Kothe, Chain configuration and flexibility gradient in phospholipid membranes. Comparison between spin-label electron spin resonance and deuteron nuclear magnetic resonance, and identification of new conformations, Biophys. J. 55 (1989) 111-123.

[22] E.C. Howard, K.M. Lindahl, C.F. Polnaszek, D.D. Thomas, Simulation of saturation transfer electron paramagnetic resonance spectra for rotational motion with restricted angular amplitude, Biophys. J. 64 (1993) 581-593.

[23] D. Marsh, A. Watts, Spin-labeling and lipid-protein interactions in membranes, in: P.C. Jost, O.H. Griffith (Eds.), Lipid-Protein Interactions, vol. 2, Wiley-Interscience, New York, 1982, pp. $53-$ 126.

[24] P. Fajer, D. Marsh, Microwave and modulation field inhomogeneities and the effect of cavity Q in saturation transfer ESR spectra. Dependence on sample size, J. Magn. Reson. 49 (1982) 212-224.

[25] D. Marsh, ESR probes for structure and dynamics of membranes, in: P.M. Bayley, R.E. Dale (Eds.), Spectroscopy and the Dynamics of Molecular Biological Systems, Academic Press, London, 1985, pp. 209-238.

[26] K. Halbach, Über eine neue Methode zur Messung von Relaxationszeiten und über den Spin von $\mathrm{Cr}^{53}$, Helv. Phys. Acta 27 (1954) 259-282.

[27] V.A. Livshits, Slow anisotropic tumbling in ESR spectra of nitroxyl radicals, J. Magn. Reson. 24 (1976) 307-313.

[28] L. Kar, E. Neyigner, J.H. Freed, Electron spin resonance and electron spin echo study of oriented multilayers of $L-\alpha$-dipalmitoylphosphatidylcholine water systems, Biophys. J. 48 (1985) 569-595.

[29] T. Páli, L.I. Horváth, D. Marsh, Continuous-wave saturation of two-component, inhomogeneously broadened, anisotropic EPR spectra, J. Magn. Reson. A101 (1993) 215-219.

[30] T.G. Castner Jr., Saturation of the paramagnetic resonance of a V center, Phys. Rev. 115 (1959) 1506-1515.

[31] J.S. Hyde, W.K. Subczynski, Spin-label oximetry, in: L.J. Berliner, J. Reuben (Eds.), Spin Labeling. Theory and Applications, Plenum Press, New York and London, 1989, pp. 399-425.

[32] V.A. Livshits, B.G. Dzikovski, D. Marsh, Oxygen permeation profile in lipid membranes: comparison with transmembrane polarity profile, Biophys. J. (2003) in press.

[33] W.K. Subczynski, J.S. Hyde, A. Kusumi, Oxygen permeability of phosphatidylcholine-cholesterol membranes, Proc. Natl. Acad. Sci. USA 86 (1989) 4474-4478.

[34] W.K. Subczynski, J.S. Hyde, A. Kusumi, Effect of alkyl chain unsaturation and cholesterol intercalation on oxygen transport in membranes - a pulse ESR spin labeling study, Biochemistry 30 (1991) 8578-8590.

[35] W.L. Hubbell, C. Altenbach, Site-directed spin-labeling of membrane proteins, in: S.H. White (Ed.), Membrane Protein Structure: Experimental Approaches, Oxford University Press, New York, 1994, pp. 224-248.

[36] C. Altenbach, D.A. Greenhalgh, H.G. Khorana, W.L. Hubbell, A collision gradient method to determine the immersion depth of nitroxides in lipid bilayers: application to spin-labeled mutants of bacteriorhodopsin, Proc. Natl. Acad. Sci. USA 91 (1994) 16671671. 